\title{
Multi-Locus Sequence Typing of Ehrlichia chaffeensis Reveals Extensive Genotypic Variation across the United States
}

\author{
Maria L. Zambrano, Christopher D. Paddock, and Sandor E. Karpathy* \\ Rickettsial Zoonoses Branch, Centers for Disease Control and Prevention, Atlanta, Georgia
}

\begin{abstract}
Ehrlichia chaffeensis causes human monocytic ehrlichiosis, and its principal vector is the Amblyomma americanum tick. The most frequently identified cases of ehrlichiosis come from the southeastern and south central states of the United States. In this study, a molecular typing system was developed that allows for the genetic differentiation of $E$. chaffeensis isolates. This multi-locus typing system included sequencing and analyzing intergenic regions ECH0033-ECH0035 and ECH0217-ECH0218, plus, variable genes variable length PCR target, 28-kDa, 120-kDa, and hemE. We examined a total of 31 unique isolates from humans and white-tailed deer, and eight DNA samples extracted from infected $A$. americanum collected from multiple states. This is the largest evaluation of $E$. chaffeensis isolates and their genotypes. Our findings show that when sequences of all six loci were concatenated and compared, the 39 samples could be separated into 23 genotypes and further grouped into six phylogenetic clades. The data in this study show no clear pattern between the geographic alignment with the genetic differentiation between the strains. As a result, this poses a challenge to understanding the spread of $E$. chaffeensis in the United States. Interestingly, our findings indicate that multiple strains from distant geographic origins share the same mutations, which suggests that the strains are being moved from one site to another by their hosts or vectors. In addition, we are seeing a northward shift in the lone star tick distribution in the United States. Last, some data also suggest minimal genetic mutations have occurred over time among strains that are within geographical proximity.
\end{abstract}

\section{INTRODUCTION}

Ehrlichia chaffeensis, a tick-borne bacterium that causes human monocytic ehrlichiosis, is transmitted to humans and various species of domesticated and wild animals by Amblyomma americanum (the lone star tick). ${ }^{1}$ Amblyomma americanum is an abundant and widely distributed humanbiting hard tick that occurs throughout a large expanse of the eastern United States. ${ }^{1,2}$ Human ehrlichiosis was first described in the United States in 1987, and the first isolation of the pathogen in cell culture was reported in $1991 .^{3-5}$ The organism was designated a new species, $E$. chaffeensis, based on its $16 \mathrm{~S}$ rRNA gene sequence, which was significantly different from those of previously described Ehrlichia species. ${ }^{4,6}$ Since then, two additional species, Ehrlichia ewingii and Ehrlichia muris euclairensis, have been identified as pathogens of humans in the United States, although infections with $E$. chaffeensis remain the most important in terms of magnitude and severity. ${ }^{7-9}$ In the United States, cases of ehrlichiosis are most frequently identified from the southeastern and central states. ${ }^{4,10-12}$ The severity of infections varies greatly, from moderately severe to fatal disease. ${ }^{13}$ Strain determinants for differences in clinical presentation are poorly understood. ${ }^{14,15}$

A few studies have examined one or two genetic loci, including the variable length PCR target (VLPT), the $120-\mathrm{kDa}$, and the $28-\mathrm{kDa}$ antigen genes to distinguish isolates of $E$. chaffeensis, and some of these investigations have identified genetic patterns among loci to suggest geographic clustering of certain strains. ${ }^{1,3,15-19}$ To more fully characterize the genetic variability of $E$. chaffeensis and identify molecular patterns that potentially correlate with geographic origin, we examined genomic DNA from 39 isolates of $E$. chaffeensis obtained from humans and white-tailed deer, as well as DNA samples extracted from infected $A$. americanum.

* Address correspondence to Sandor E. Karpathy, Rickettsial Zoonoses Branch, Centers for Disease Control and Prevention, 1600 Clifton Rd. NE, Atlanta, GA 30333. E-mail: evu2@cdc.gov

\section{MATERIALS AND METHODS}

Isolates and DNA extraction. Thirty isolates of $E$. chaffeensis, obtained from humans $(n=28)$ or white-tailed deer $(n=2)$ during 1991-2013, were included in the analysis (Table 1). These isolates originated from a minimum of 22 distinct locations in nine southeastern and Midwestern states. $3,5,18,20-27$ All $E$. chaffeensis isolates were cultured in continuous canine macrophage $\mathrm{DH} 82$ cells maintained at $37^{\circ} \mathrm{C}$ in a $5 \% \mathrm{CO}_{2}$ atmosphere using minimal essential medium (Gibco) supplemented with $10 \%$ heat-inactivated fetal bovine serum, $0.1 \mathrm{~mm}$ MEM nonessential amino acids (Gibco), $0.1 \mathrm{~mm}$ HEPES (Gibco), $2 \mathrm{~mm}$ L-glutamine (Gibco), and $10 \mathrm{~mm}$ sodium pyruvate. $^{3,28}$ Infected cultures were monitored for Ehrlichia morulae using cytospin slide preparations stained with DiffQuick. When the E. chaffeensis cultures were harvested for cryopreservation, a $0.5-\mathrm{mL}$ aliquot was set aside for DNA extraction using the KingFisher Cell \& Tissue DNA kit (Thermo Fisher Scientific, Waltham, MA) and KingFisher $\mathrm{mL}$ magnetic particle processor. In addition, DNA extracted from nine, $E$. chaffeensis-infected $A$. americanum ticks, collected from four separate locations in New York and Connecticut, were also used in the analyses. DNA from the infected $A$. americanum ticks was extracted using the Qiagen DNeasy Blood and Tissue Kit (Qiagen, Germantown, MD).

Selection of loci. A subset of four isolates that originated from widely separated geographical areas (Arkansas ${ }^{\top}$, Heartland, Jax, and Wakulla) was selected to evaluate candidate genes and intergenic regions for nucleotide differences. Six loci were selected for analysis: VLPT, 120-kDa protein gene, 28-kDa protein gene, hemE, and intergenic regions between $\mathrm{ECH} 0217-0218$ and $\mathrm{ECH0033-ECH0035.} \mathrm{The}$ VLPT, $120-\mathrm{kDa}$, and 28-kDa genes have been used in previous studies to distinguish between a smaller set of $E$. chaffeensis isolates. ${ }^{1,17,19}$ In addition, intergenic regions between ECH02170218 and $\mathrm{ECH0033-ECH0035} \mathrm{were} \mathrm{identified} \mathrm{because} \mathrm{of}$ differences found in the whole genome alignments between the following isolates: Arkansas ${ }^{\top}$, Heartland, Jax, 
TABLE 1

Ehrlichia chaffeensis isolates and DNA samples from Amblyomma americanum ticks used in this study

\begin{tabular}{|c|c|c|c|c|}
\hline Isolate or tick DNA & Source & Year & Location & Reference \\
\hline Arkansas $^{\top}$ & Human & 1991 & Sebastian County, Arkansas & 3 \\
\hline 91HE17 & Human & 1991 & Arkansas & 25 \\
\hline $\mathrm{MO}-18$ & Human & 2013 & Holt County, Missouri & This study \\
\hline Heartland & Human & 1999 & Nebraska & 30 \\
\hline Jax & Human & 1996 & Nassau County, Florida & 26 \\
\hline MO-19 & Human & 2013 & Nodaway County, Missouri & This study \\
\hline Liberty & Human & 1998 & Liberty County, Florida & \\
\hline $\mathrm{MO}-13$ & Human & 2009 & Andrew County, Missouri & This study \\
\hline $\mathrm{MO}-17$ & Human & 2011 & Doniphan County, Kansas & This study \\
\hline $\mathrm{MO}-3$ & Human & 2009 & Missouri & This study \\
\hline $\mathrm{MO}-6$ & Human & 2009 & Missouri & This study \\
\hline Osceola & Human & 1997 & Baker County, Florida & 4 \\
\hline Sapulpa & Human & 1995 & Tulsa County, Oklahoma & 16 \\
\hline St. Vincent & Human & 1996 & Bibb County, Georgia & 26 \\
\hline V1 & Human & 1998 & Tennessee or Kentucky & 27 \\
\hline V3 & Human & 1998 & Tennessee or Kentucky & 27 \\
\hline V4 & Human & 1998 & Tennessee or Kentucky & 27 \\
\hline V5 & Human & 1998 & Tennessee or Kentucky & 27 \\
\hline V6 & Human & 1998 & Tennessee or Kentucky & 27 \\
\hline V7 & Human & 1998 & Tennessee or Kentucky & 27 \\
\hline V8 & Human & 1999 & Unknown & 20 \\
\hline V9 & Human & 1999 & Unknown & 20 \\
\hline V10 & Human & 1999 & Unknown & 22 \\
\hline V19 & Human & 1999 & Unknown & This study \\
\hline Wakulla & Human & 1997 & Wakulla County, Florida & 4 \\
\hline West paces & Human & 1998 & Tennessee & 4 \\
\hline CT17-107 & A. americanum & 2017 & Fairfield County, Connecticut & 24 \\
\hline CT17-121 & A. americanum & 2017 & Fairfield County, Connecticut & 24 \\
\hline CT17-126 & A. americanum & 2017 & Fairfield County, Connecticut & 24 \\
\hline CT17-143 & A. americanum & 2017 & Fairfield County, Connecticut & 24 \\
\hline CT17-174 & A. americanum & 2017 & Fairfield County, Connecticut & 24 \\
\hline 2018NY-Aa95m & A. americanum & 2018 & Suffolk County, New York & This study \\
\hline 2018NY-Aa140m & A. americanum & 2018 & Suffolk County, New York & This study \\
\hline 2018NY-Aa171m & A. americanum & 2018 & Suffolk County, New York & This study \\
\hline Lithonia & Human & 2000 & DeKalb County, Georgia & \\
\hline Chattanooga & Human & 2000 & Tennessee & 30 \\
\hline Mitch & O. virginianus & 1995-1996 & Georgia & 23 \\
\hline Dave & O. virginianus & 1995-1996 & Georgia & 23 \\
\hline
\end{tabular}

Liberty, Osceola, Sapulpa, St. Vincent, Wakulla, and West Paces.

Molecular analyses. Standard PCR reactions were set up for six loci. Each PCR reaction consisted of $2 \mu \mathrm{L}$ DNA template, $10 \mu \mathrm{L}$ of Taq PCR Master Mix (QIAGEN, Valencia CA), and each primer at a final concentration of $1 \mu \mathrm{M}$ in a final reaction volume of $20 \mu \mathrm{L}$. After a 5-minute denaturation at $95^{\circ} \mathrm{C}$, each reaction underwent 40 cycles of a 30 -second denaturation at $95^{\circ} \mathrm{C}$, a 30 -second annealing incubation (Table 2), and a 1-minute extension at $72^{\circ} \mathrm{C}$ for the hemE gene and intergenic regions ECH0217-0218 and ECH0033ECH0035 (Table 2). This was followed by a final 10-minute extension at $72^{\circ} \mathrm{C}$. PCR cycling conditions for the VLPT, $120 \mathrm{kDa}$, and $28 \mathrm{kDa}$ protein genes were used as previously described. ${ }^{1,17,19}$ Amplicons were resolved in a 1\% agarose gel and purified using the Promega Wizard SV gel extraction kit and PCR Clean-Up System (Promega, Madison WI) following the manufacturer's protocol.

Sequencing and phylogenetic analyses. One microliter of the purified PCR product was sequenced using a BigDye Terminator v. 3.1 cycle sequencing kit (Applied Biosystems, Foster City, CA) on an ABI 3500 genetic analyzer. Each PCR amplicon was sequenced in both directions, and sequence reads were assembled using Geneious R11 (Biomatters Ltd.,
Auckland, New Zealand). MEGA7 was used to create DNA alignments for each locus using CLUSTAL W. ${ }^{29}$ For the concatenated alignment, the loci were concatenated in the following order: ECH0033-ECH0035, ECH0217-ECH0218, hemE, VLPT, 28-kDa, and 120-kDa. MEGA7 was used to perform the phylogenetic analysis, a neighbor-joining tree was constructed using the maximum composite likelihood JukesCantor model, and 1,000 bootstrap replicates were used to estimate the likelihood of the tree.

\section{RESULTS}

ECH0033-ECH0035. The primers for this locus amplified a 711-bp fragment of the intergenic region between two hypothetical proteins of unknown function (ECH0033-ECH0035). Five single nucleotide polymorphisms (SNPs) were identified within the amplicons generated by the ECH0033-ECH0035 primer pair (Figure 1), resulting in the identification of seven genotypes. The Lithonia isolate and tick DNA 2018NYAa140M are $100 \%$ identical to Arkansas ${ }^{\top}$ (genotype I). When compared with genotype I, isolates V3, V8, V9, Chattanooga, Dave, Heartland, Sapulpa, and West Paces have a single SNP (C to T transition) at nucleotide 134 and comprise genotype II. Isolates V7, Liberty, and tick DNA samples CT17-121, and 


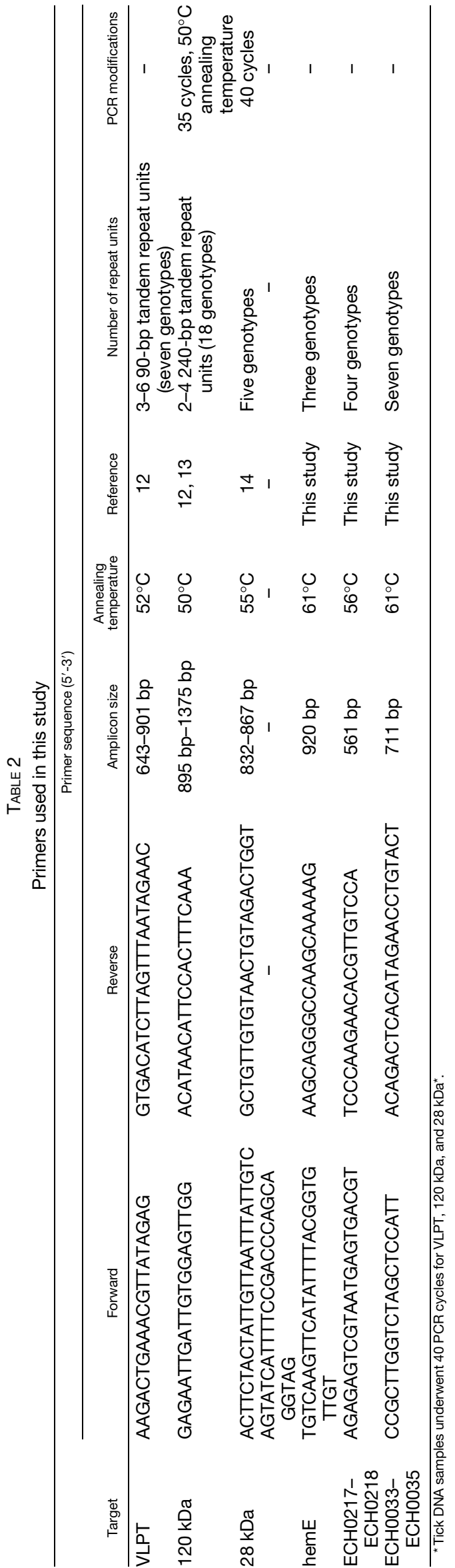

CT17-174 make up genotype III. Compared with strain Arkansas $^{\top}$, these isolates contain a single SNP at nucleotide position 356 ( $\mathrm{C}$ to $\mathrm{T}$ transition). Eight isolates (MO-3, MO-6, $\mathrm{MO}-17, \mathrm{MO}-18, \mathrm{MO}-19, \mathrm{~V} 6, \mathrm{~V} 10$, and V19) are included in genotype IV. These isolates have two SNPs compared with genotype I, they are located at nucleotide positions 446 ( $T$ to $C$ transition) and 476 ( $G$ to $A$ transition). Genotype $V$ contains isolates V4, V4C, Jax, Osceola, and Wakulla, and tick DNAs 2018NY-Aa95M and 2018NY-Aa171M. These strains are identical with strain Arkansas $^{\top}$, except that they share the SNP at nucleotide 476 with genotype IV. The genotype $\mathrm{VI}$ isolates (V1, V5, 91HE17, MO-13, Mitch, and St. Vincent) share a single SNP with genotype IV at nucleotide position 446, whereas genotype VII, represented by tick DNAs CT17-107, CT17-126, and CT17-143, contains two SNPs at nucleotide positions 356 (shared with genotype III) and 659 (a unique A to G transition) when compared with genotype I.

ECH0217-ECH0218. These primers amplified a 561-bp region of the intergenic region between a putative methylthiotransferase (ECH0217) and a predicted thioredoxin (ECH0218). Three SNPs were detected in the ECH0217$\mathrm{ECH} 0218$ intergenic region to that created four distinct genotypes (Figure 1). DNA from tick 2018NY-Aa171M, and isolates Lithonia, Mitch, V1, and V5 are 100\% identical to Arkansas $^{\top}$ and comprise genotype I. Isolates V3, V8, V9, Chattanooga, Heartland, Sapulpa, St. Vincent, and West Paces, and DNA samples from ticks CT17-107, CT17-121, CT17-126, CT17-143, CT17-174, and 2018NY-Aa140M contain an SNP (G to A transition) at position 132 and form genotype II. Genotype III isolates (V7, 91HE17, MO-13, Dave, Osceola, and Wakulla) also have a single SNP located at nucleotide position 198 ( $T$ to $\mathrm{C}$ transition). The isolates that constitute genotype IV have two SNPs present at nucleotide positions 198 ( $T$ to $C$ transition) and 458 ( $T$ to $C$ transition) and include V4, V4C, V6, V10, V19, MO-3, MO-6, MO-17, MO-18, MO-19, Jax, and Liberty, and tick DNA 2018NY-Aa95M.

hemE protein gene. These primers amplified a 920-bp region of hemE, a uroporphyrinogen decarboxylase which is a component in heme biosynthesis. ${ }^{14}$ The hemE amplicons had nine SNPs producing a total of three distinct genotypes (Figure 1). Isolates V1, V5, Dave, Lithonia, Mitch, and Osceola, and tick DNA 2018NY-Aa95M were all identical to Arkansas ${ }^{\top}$ and comprise genotype I. The Wakulla isolate had a unique genotype, with nine SNPs at nucleotide positions 11 (G to A transition), 28 (C to $T$ transition), 62 (T to $\mathrm{C}$ transition), 173 (A to G transition), 203 (G to A transition), 306 (T to $C$ transition), 797 ( $G$ to $A$ transition), 884 ( $C$ to $T$ transition), and 901 ( $C$ to $A$ transversion). The remaining isolates and tick DNA samples were identical to each other and comprise genotype III. This genotype contains the SNPs found in genotype II at nucleotides 797,884 , and 901 , but is identical to genotype I at all other positions.

28-kDa protein gene. The primers for this locus amplified an $832-$ to $867-b p$ (isolate dependent) region of the $28-\mathrm{kDa}$ outer membrane protein, which is involved in disulfide-bond cross-linking of the outer membrane of Ehrlichia. ${ }^{1}$ Two hundred two SNPs and 12 insertion/deletion (INDEL) events were identified in the 28-kDa amplicons, which created a total of five distinct genotypes (Figure 1). Isolates identical to Arkansas ${ }^{\top}$ (genotype I) include VI, V5, Dave, Lithonia, Mitch, and Osceola. Genotype II isolates comprised V4, V4C, V7, MO-13, 91HE17, Liberty, and Jax. In comparison to Arkansas ${ }^{\top}$, genotype II 
ECH0033-ECH0035

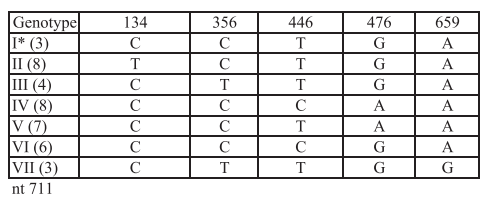

VLPT

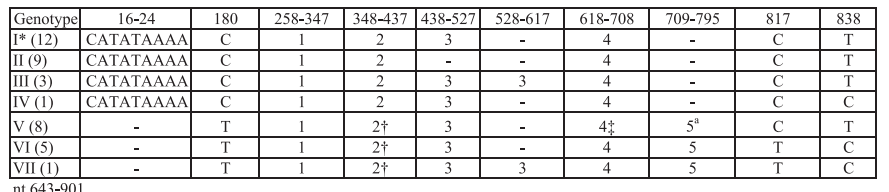

ECH0217-ECH0218

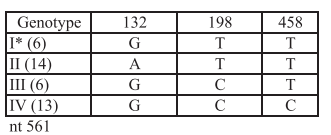

hemE

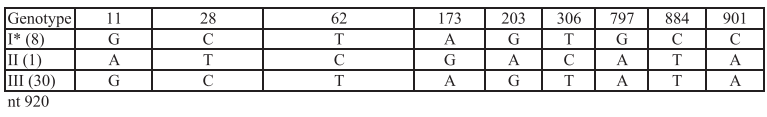

$28 \mathrm{kD}=$

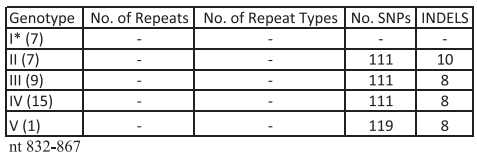

$120 \mathrm{kDa}$

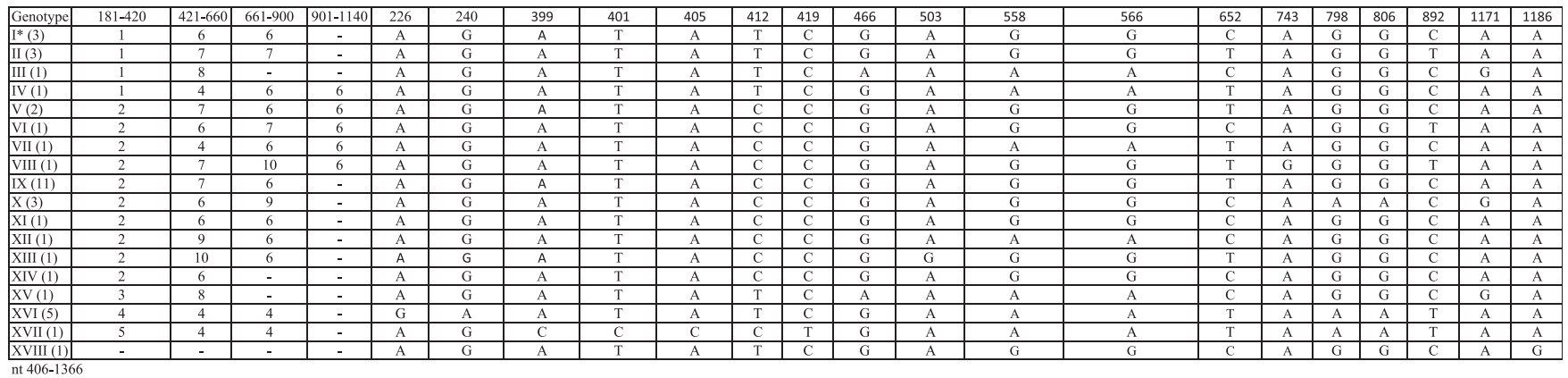

FIGURE 1. Genotypes of sequenced intergenic regions and outer membrane proteins. Each table shows individual genotypes identified for each locus. The numbers in parentheses indicate the number of isolates in each genotype. The total number of nucleotides in the consensus sequence of each locus is shown under the table, and the nucleotide positions indicated are relative to the aligned consensus sequence. ${ }^{*}$ Type strain for this study is Arkansas. Note: VLPT sequence units are 90-bp and found in consensus nucleotides $258-347,348-437,438-527,528-617,618-708$, and 709-795; sequence unit types are designated numbers 1-5. Note: $120 \mathrm{kDa}$ sequence units are 240-bp and found in consensus nucleotides 181-420, 421-660, 661-900, and 901-1,140; sequence unit types are designated numbers 1-10.†single nucleotide polymorphisms at nucleotide position 420 (A to $\mathrm{G}$ transition). $¥ 18$-bp deletion at consensus nucleotides $690-707 .{ }^{a} 71$-bp deletion at consensus nucleotides $709-779$. ${ }^{b} 16-b p$ deletion at consensus nucleotides $790-795$.

isolates have 104 SNPs and 10 INDELS events. Genotype III isolates (V3, V8, V9, Chattanooga, Heartland, Sapulpa, St. Vincent, and West Paces, and tick DNA 2018NY-Aa140M) exhibit 116 SNPs and eight INDEL events. Genotype IV (isolates V6, V10, V19, MO-3, MO-6, MO-17, MO-18, MO-19, and Wakulla, and tick DNAs 2018NY-Aa171M, CT17-107, CT17121, CT17-126, CT17-142, and CT17-174) and genotype V (tick DNA 2018NY-Aa95M) had 112 and 118 SNPs, respectively. Furthermore, genotype IV and V isolates share the same INDEL events at nucleotide positions 13, 85, 97, 110, 121-135, 223-228, 534-548 (genotype I Indel 534-545), 558-560 as genotype I.

120-kDa protein gene. The primers for this locus amplified a fragment of the $120-\mathrm{kDa}$ antigen gene between 406 and $1,366 \mathrm{bp}$ in length, depending on the isolate. This immunodominant protein is mainly expressed on dense-core stages of E. chaffeensis. ${ }^{17}$ Expression of this gene in Escherichia coli causes attachment and internalization by Vero cells, suggesting an important role for this protein in the attachment and invasion of host cells by $E$. chaffeensis. ${ }^{17}$ The amplicons produced by the 120-kDa primers contained two to four, 240bp repeat units, as previously described. ${ }^{17,19}$ The 240 -bp repeat units identified were found at consensus nucleotides 181-420, 421-660, 661-900, and 901-1,140 (Figure 1). In addition, a total of 18 SNPs were found among the 18 genotypes identified (Figure 1). Isolates V1 and Mitch are identical to Arkansas $^{\top}$ (Genotype I). These isolates have three repeat units with unique repeat unit types and sequence profile 1, 6, and 6 (Figure 1). Other genotypes that have three repeat units with distinct sequence profiles include genotypes II (isolates MO-17, MO-18, and MO-19), IX (V3, V8, V9, Chattanooga, Heartland, Liberty, Sapulpa, and West Paces, and tick DNAs CT17-107, CT17-126, and CT17-174), X (isolates Osceola, and St. Vincent, and tick DNA 2018NY-Aa140M), XI (isolate MO-13), XII (isolate 91HE17), XIII (tick DNA CT17-143), XVI (isolates MO-3, MO-6, V6, V10, and V19), and XVII (tick DNA 2018 NY-171m) (Figure 1). They also share the same INDEL between nucleotide positions 901-1,140 (Figure 1). In comparison to Arkansas ${ }^{\top}$, the isolates that comprise genotypes IV (Wakulla), V (V4C, Jax), and VII (V7), and tick DNAs comprising genotypes VI (2018-NYAa95M) and VIII (CT17-121) share four repeat units, again, with their own distinct repeat unit types and profiles (Figure 1). Furthermore, isolates Lithonia (genotype III), V4 (genotype XIV), and V5 (genotype XV) have only two repeat units and share the same INDEL events at nucleotide positions 421-660 and 661-900 (Figure 1). In contrast to all other strains, Wakulla (genotype XVIII) was the only isolate that had no repeat units present (Figure 1 ). In conclusion, there was a total of 10 different 240-bp imperfect repeat unit types identified within the four $240-b p$ repeat regions which produced 18 genotypes (Figure 1).

Variable length PCR target protein gene. The primers for the locus amplified a 643- to 901-bp region of the VLPT protein. This gene has been shown to both be a sensitive and specific marker for $E$. chaffeensis and to allow for limited differentiation between strains of $E$. chaffeensis. ${ }^{4}$ Four SNPs, six INDELS, up to six imperfect repeat units (three to six repeat units, depending on the isolate), and five versions of the repeat 


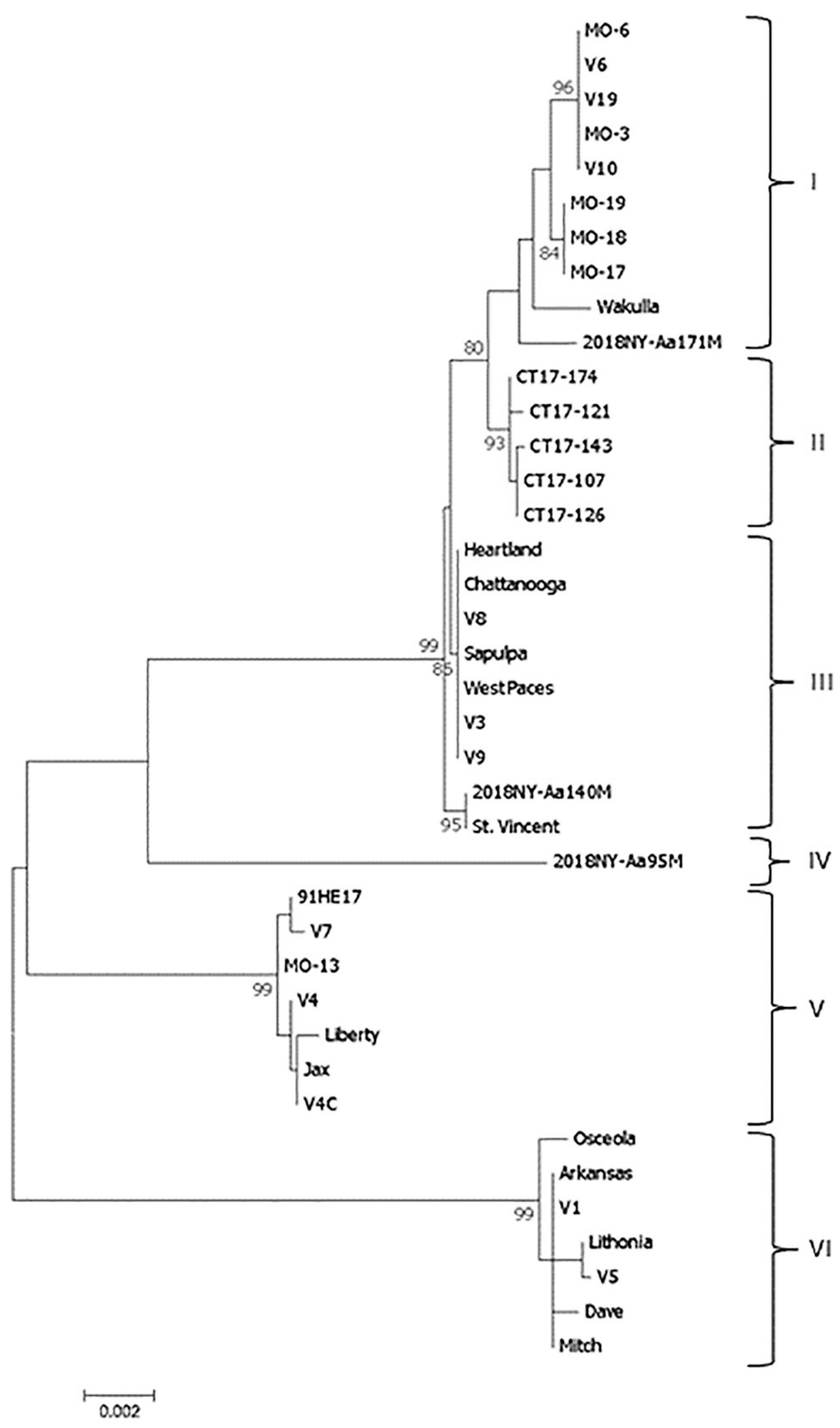

FIGURE 2. Phylogenetic associations of Ehrlichia chaffeensis tick DNAs and isolates. Maximum composite likelihood Jukes-Cantor model phylogenetic relationships of $E$. chaffeensis are based on the concatenated sequences six loci (ECH0033-ECH0035, ECH0217-ECH0218, hemE, VLPT, 28-kDa, and 120-kDa). Numbers at the nodes are bootstrap values based on 1,000 bootstrap replicates. Only bootstrap values $>70 \%$ are shown. 
sequence were detected in the VLPT amplicons that collectively created seven distinct genotypes (Figure 1). Four SNPs were detected at nucleotide positions 180 (C to $\mathrm{T}$ transition), 420 (A to $\mathrm{G}$ transition), 817 (C to T transition), and 838 (T to C transition). As previously described, VLPT amplicons also contained three to six repeat units at nucleotide positions 258-347, 348-437, 438-527, 528-617, 618-708, and 709-795, respectively. ${ }^{4}$ Isolates V1, V4, V4C, V5, Dave, Jax, Lithonia, Mitch, and Osceola, and tick DNA specimens 2018NY-Aa95M and 2018NY-Aa171M are 100\% identical to Arkansas $^{\top}$ (Genotype I) and consist of four repeat units with repeat profiles 1, 2, 3, and 4 (Figure 1). Isolates V3, V8, V9, Heartland, Sapulpa, St. Vincent, 91HE17, and Chattanooga, and tick DNA 2018NY-Aa140M, comprising genotype II, have a ninety-bp deletion at consensus nucleotides 438-527 when compared with Arkansas ${ }^{\top}$. The three repeat units have a repeat profile of 1,2 , and 4 . Genotype III consists of the isolates MO-13, V7, and West Paces, and has five repeat units with the profiles $1,2,3,3$, and 4 . A single SNP was identified at nucleotide position 838 ( $T$ to $\mathrm{C}$ transition) that differentiates isolate Liberty (Genotype IV) from strain Arkansas $^{\top}$ and genotype I. Isolates MO-3, MO-6, MO-17, MO-18, MO-19, V6, V10, V19, comprising genotype V, contain a unique 90-bp deletion between nucleotides 690-779 (in repeats four and five). This genotype also contains a 9-bp deletion of nucleotides 16-24. In addition, this genotype contains an SNP at nucleotide 180 (C to $T$ transition) and an additional SNP in repeat two at nucleotide 420 (A to G transition). These isolates possess five repeat units with a repeat profile of $1,2,3,4$, and 5. Tick DNA samples CT17-107, CT17-121, CT17-126, CT17-143, and CT 17-174 comprise genotype VI. This genotype shares the deletion of nucleotides 16-24 and the SNP at position 180 with genotype $V$ and contains full-length versions of repeats four and five. In addition, there is a SNP at position 817 (C to T transition), and this genotype shares the SNP at nucleotide 838 with genotype IV. The isolate Wakulla has a unique genotype (genotype VII). This isolate is identical to genotype VI, with the exception that it shares a second copy of repeat three with genotype III. Thus, this isolate contains six repeat units with the repeat pattern 1, 2, 3, 3, 4, and 5.

Phylogenetic analysis. Concatenation of the six loci separated the 30 isolates and nine tick DNA samples into 23 genotypes comprising six phylogenetic clades (Figure 2). MEGA7 was used to perform the phylogenetic analysis, a neighbor-joining tree was constructed using the maximum composite likelihood Jukes-Cantor model, and 1,000 bootstrap replicates were used to estimate the likelihood of the tree. Clades were established based on bootstrap values $>70$. Any bootstrap value $<70$ was considered statistically nonsignificant to designating a distinct clade. Clade I contained four closely related genotypes, comprising five isolates from Missouri, one isolate from Florida, a tick DNA sample from Suffolk County, New York, and three isolates from Tennessee/Kentucky. Clade II comprised all five tick DNA samples from Connecticut in four closely related genotypes. Clade III consists of nine isolates in two closely related genotypes. The first genotype from Clade III consists of an isolate from Florida and one tick DNA sample from Suffolk County, New York. The second genotype in Clade III consists of isolates from Nebraska, Georgia, Oklahoma, and Tennessee/Kentucky. Clade IV consists of a single tick DNA sample from Suffolk County, New York. There are seven isolates in six closely related genotypes coming from geographic locations such as Arkansas, Missouri, Florida, and Tennessee/Kentucky in clade V. Finally, clade VI has seven isolates with six closely related genotypes from Florida, Tennessee, Arkansas, and Georgia.

\section{DISCUSSION}

In this study, a multi-locus typing system was developed that allows for the genetic differentiation of $E$. chaffeensis. To our knowledge, this represents the largest genotypic evaluation of this tick-borne pathogen and included 30 unique isolates and nine tick-associated DNA specimens from 11 U.S. states. The analysis identified a total of 515 individual mutations (234 SNPs and 21 INDELs) among these six targets that generated 23 different genotypes that could be grouped into six different phylogenetic clades (Figure 2). The 120-kDa locus presents the largest number of $E$. chaffeensis genotypes (18), whereas the VLPT locus exhibits seven genotypes among the isolates. Moreover, the $28-\mathrm{kDa}$ locus shows the greatest number of SNPs and INDEL events between the isolates. None of the other loci (hemE, ECH0033-ECH0034, and ECH0217-ECH0218) revealed repeat sequences or INDELs. What differentiated the strains were the presence of 9,5 , and 3 SNPs as different nucleotide positions, respectively. The data in this study suggest that protein genes VLPT, 120$\mathrm{kDa}, 28-\mathrm{kDa}$, and hemE can be used as dependable markers to distinguish among several isolates of $E$. chaffeensis, as well as the intergenic regions $\mathrm{ECH} 0033-\mathrm{ECH} 0034$ and ECH0217-ECH0218.

To our knowledge, this study represents the largest analysis of genetic diversity in $E$. chaffeensis, both in the number of isolates or DNA samples and in the number of genetic loci analyzed. Although our study generally agrees with the results of previous studies, it provides increased granularity into the genetic diversity of this pathogen and allows for additional separation of isolates and DNA samples. By using only the $28-\mathrm{kDa}$ protein gene, $\mathrm{Yu}$ et al. were able to separate five isolates into three genetic groups. ${ }^{1}$ Our analysis identified the same three groupings, but also identified an additional three clades. Also, using the $28-\mathrm{kDa}$ gene, Cheng et al. ${ }^{30}$ analyzed 10 isolates and found the same three groups. Our analysis characterized three additional clades and divided one of their groups (group 3) into two distinct clades (clade I and clade III). Finally, Sumner et al. ${ }^{4}$ reported 12 genotypes when analyzing the nucleotide sequences of the VLPT gene from nine isolates, seven positive patient DNA samples, and eight positive tick pool DNA samples. Our analysis nearly doubled the number of genotypes.

Our analyses did not identify specific associations between disease severity and various genotypes or clades. Among the four clades with representation by isolates of human origin, three (clades III, V, and VI) contained E. chaffeensis genotypes cultivated from patients with fatal disease (i.e., Jax, St. Vincent, Osceola, and Chattanooga) ${ }^{26}$ or life-threatening infection (i.e., Sapulpa and $91 \mathrm{HE} 17),{ }^{16,25}$ as well as isolates from patients with varying degrees of disease severity who survived their illnesses (i.e., Arkansas and V1-V7). ${ }^{3,27}$ Similarly, clade I contained isolates from patients with disease of sufficient severity to require hospitalization (i.e., Wakulla), but who recovered 
fully because of early diagnosis and appropriate antibiotic therapy. ${ }^{31}$ In this context, it is possible that most or possibly all genotypes of $E$. chaffeensis are capable of causing severe or fatal disease in a human host. Multiple other intrinsic and extrinsic factors, including the age and underling immune status of the host, and the timeliness of diagnosis and treatment, are crucial considerations assessing patient outcomes following infection. ${ }^{27,31}$ With a few exceptions, no clear patterns emerged from our analysis that predicted geographical distribution based on genotype. Surprisingly, we found that multiple isolates and DNA specimens shared similar or identical genotypes, despite widely separated geographical origins. Also surprising was the identification of extensive genetic variability among samples of $E$. chaffeensis originating from relatively close geographical regions. For example, clade VI comprised seven isolates from Florida, Georgia, Tennessee or Kentucky, and Arkansas. These seven isolates have closely related genotypes, with three isolates (from Arkansas, Georgia, and Tennessee or Kentucky) sharing an identical genotype. Yet, the seven isolates from the adjacent states of Tennessee and Kentucky contain seven different genotypes that group into five separate clades, and the four isolates from Florida contain four genotypes grouped in four clades. Likewise, the five DNA samples from Connecticut have four closely related genotypes that group into a single clade (Clade II), whereas the three DNA samples from New York have three very different genotypes, each grouping into different clades (clade I, clade III, and clade IV).

The broad geographic distribution of $E$. chaffeensis strains that share similar genotypes may represent the introduction of a new lineage into a geographic area due to the migration of vertebrate hosts. Amblyomma americanum (lone star) ticks are the principal vectors of $E$. chaffeensis. ${ }^{2}$ These ticks are the most abundant and aggressive human-biting tick in the southeastern United States, and are most commonly associated with white-tailed deer and wild turkeys. ${ }^{32,33}$ However, they feed on a wide range of mammals including cattle, horses, cats, coyotes, dogs, foxes, opossums, rabbits, raccoons, rodents, and squirrels as well as birds (i.e., gallinaceous and passerine birds). ${ }^{32}$ Most importantly, white-tailed deer, wild turkeys, and coyotes have played an important role as hosts for all life stages of the lone star ticks. ${ }^{21}$ The emergence of Amblyomma-associated ehrlichiosis has been influenced in part by the explosive growth of various wildlife species, especially white-tailed deer, wild turkeys, and coyotes. ${ }^{21}$ For instance, coyotes have expanded their range throughout North America from the Great Plains and western United States to the southeast. ${ }^{21}$ These carnivores exist in most habitats and have become established in suburban and urban locations which increase the potential for infected lone star ticks to be seeded into the peri-domestic environment. ${ }^{21}$ Last, as the wild turkey populations have increased throughout their historic geographic range (New York to the north and Kansas to the west), they are credited with reintroducing and increasing population densities of $A$. americanum. ${ }^{21}$ However, although increases in the populations of white-tailed deer, coyotes, and wild turkeys may explain local increases in $E$. chaffeensis-infected ticks, their relatively small home ranges (182-416 ha, 3,130-6,870 ha, and 1,295-3,514 ha, respectively) would not explain the existence of closely related or identical genotypes of $E$. chaffeensis across large geographic distances, especially given the genetic diversity seen in localized areas. ${ }^{34-36}$

The distribution of $E$. chaffeensis across large geographical distances by highly mobile species such as birds could explain the occurrence of identical genotypes in distant locations. Ehrlichial DNA, including that of $E$. chaffeensis, has been detected in the blood of various avian species including the northern crested caracaras (Caracara cheriway), the American kestrel (Falco sparverius), and the black vulture (Coragyps atratus). ${ }^{37,38}$ In this context, birds could serve as amplifying hosts for the bacteria, providing a source of infection for naive ticks as well as the distribution of bacteria across broad geographic regions. ${ }^{38-40}$ Indeed, one study investigating the tick species found on birds in the state of Georgia reported that the predominate tick identified was $A$. americanum, the primary vector of $E$. chaffeensis. ${ }^{41}$ In addition, ehrlichial DNA has been detected in nymphal stage lone star ticks removed from multiple avian species, including the pale thrush (Turdus pallidus), the chaffinch (Fringilla coelebs), the common blackbird (Tardus merula), the brambling (Fringilla montifringilla), the Eurasian wren (Troglodytes troglodytes), and the willow warbler (Phylloscopus trochilus). ${ }^{42,43}$

The absence of correlation among various genotypes and geographical origins is a notable aspect of this study. The only clade represented by a single source is clade II. These ticks were collected on a small island off the coast of Connecticut and could represent a geographically isolated population. ${ }^{24}$ Although most of the isolates in this study originated from infections of humans, no genetic distinctions were identified between isolates of human origin and those directly from white-tailed deer or tick DNA. Future studies are needed to determine if any of the genetic differentiations correlate with differences in virulence.

Received October 16, 2020. Accepted for publication January 6, 2021. Published online March 1, 2021.

Acknowledgments: We thank Jere McBride (University of Texas Medical Branch at Galveston), Roman Ganta (Kansas State University), Uli Munderloh (University of Minnesota), Michael Yabsley (University of Georgia), and William Nicholson (CDC) for providing isolates of $E$. chaffeensis, and Kirby Stafford (The Connecticut Agricultural Research Station) and Moses Cucura (Suffolk County Vector Control) for providing $E$. chaffeensis-infected specimens of $A$. americanum used in this study.

Disclaimer: The findings and conclusions in this article are those of the authors and do not necessarily represent the official position of the CDC.

Authors' addresses: Maria L. Zambrano, Christopher D. Paddock, and Sandor E. Karpathy, Rickettsial Zoonoses Branch, Centers for Disease Control and Prevention, Atlanta, GA, E-mails: daq6@cdc.gov, cdp9@cdc.gov, and evu2@cdc.gov.

\section{REFERENCES}

1. Yu XJ, McBride JW, Walker DH, 1999. Genetic diversity of the 28kilodalton outer membrane protein gene in human isolates of Ehrlichia chaffeensis. J Clin Microbiol 37: 1137-1143.

2. Anderson BE, Sims KG, Olson JG, Childs JE, Piesman JF, Happ CM, Maupin GO, Johnson BJ, 1993. Amblyomma americanum: a potential vector of human ehrlichiosis. Am J Trop Med Hygiene 49: 239-244. 
3. Dawson JE, Anderson BE, Fishbein DB, Sanchez JL, Goldsmith CS, Wilson KH, Duntley CW, 1991. Isolation and characterization of an Ehrlichia sp. from a patient diagnosed with human ehrlichiosis. J Clin Microbiol 29: 2741-2745.

4. Sumner JW, Childs JE, Paddock CD, 1999. Molecular cloning and characterization of the Ehrlichia chaffeensis variable-length PCR target: an antigen-expressing gene that exhibits interstrain variation. J Clin Microbiol 37: 1447-1453.

5. Maeda K, Markowitz N, Hawley RC, Ristic M, Cox D, McDade JE, 1987. Human infection with Ehrlichia canis, a leukocytic Rickettsia. New Engl J Med 316: 853-856.

6. Anderson BE, Dawson JE, Jones DC, Wilson KH, 1991. Ehrlichia chaffeensis, a new species associated with human ehrlichiosis. $J$ Clin Microbiol 29: 2838-2842.

7. Pritt $\mathrm{B}$ et al., 2011. Emergence of a new pathogenic Ehrlichia species, Wisconsin and Minnesota, 2009. New Engl J Med 365: 422-429.

8. Pritt BS et al., 2017. Proposal to reclassify Ehrlichia muris as Ehrlichia muris subsp. muris subsp. nov. and description of Ehrlichia muris subsp. eauclairensis subsp. nov., a newly recognized tick-borne pathogen of humans. Int J Syst Evol Microbiol 67: 2121-2126.

9. Anderson BE, Greene CE, Jones DC, Dawson JE, 1992. Ehrlichia ewingii sp. nov., the etiologic agent of canine granulocytic ehrlichiosis. Int $J$ Syst Bacteriol 42: 299-302.

10. Eng TR, Harkess JR, Fishbein DB, Dawson JE, Greene CN, Redus MA, Satalowich FT, 1990. Epidemiologic, clinical, and laboratory findings of human ehrlichiosis in the United States, 1988. J Am Med Assoc 264: 2251-2258.

11. Fishbein DB, Kemp A, Dawson JE, Greene NR, Redus MA, Fields $\mathrm{DH}, 1989$. Human ehrlichiosis: prospective active surveillance in febrile hospitalized patients. J Infect Dis 160: 803-809.

12. Walker DH, Dumler JS, 1996. Emergence of the ehrlichioses as human health problems. Emerg Infect Dis 2: 18-29.

13. Biggs HM et al., 2016. Diagnosis and management of tickborne rickettsial diseases: Rocky Mountain spotted fever and other spotted fever group rickettsioses, ehrlichioses, and anaplasmosis- United States. MMWR Recom Rep 65: 1-45.

14. Miura K, Rikihisa Y, 2007. Virulence potential of Ehrlichia chaffeensis strains of distinct genome sequences. Infect Immun 75: 3604-3613.

15. Paddock CD, Childs JE, 2003. Ehrlichia chaffeensis: a prototypical emerging pathogen. Clin Microbiol Rev 16: 37-64.

16. Chen SM, Yu XJ, Popov VL, Westerman EL, Hamilton FG, Walker DH, 1997. Genetic and antigenic diversity of Ehrlichia chaffeensis: comparative analysis of a novel human strain from Oklahoma and previously isolated strains. J Infect Dis 175: 856-863.

17. Yabsley MJ, Little SE, Sims EJ, Dugan VG, Stallknecht DE, Davidson WR, 2003. Molecular variation in the variable-length PCR target and 120-kilodalton antigen genes of Ehrlichia chaffeensis from white-tailed deer (Odocoileus virginianus). J Clin Microbiol 41: 5202-5206.

18. Stromdahl EY, Randolph MP, O’Brien JJ, Gutierrez AG, 2000. Ehrlichia chaffeensis (Rickettsiales: Ehrlichiae) infection in Amblyomma americanum (Acari: Ixodidae) at aberdeen proving ground, Maryland. J Med Entomol 37: 349-356.

19. Yu XJ, Crocquet-Valdes $P$, Walker DH, 1997. Cloning and sequencing of the gene for a 120-kDa immunodominant protein of Ehrlichia chaffeensis. Gene 184: 149-154.

20. Long SW, Zhang XF, Qi H, Standaert S, Walker DH, Yu XJ, 2002. Antigenic variation of Ehrlichia chaffeensis resulting from differential expression of the 28-kilodalton protein gene family. Infect Immun 70: 1824-1831.

21. Childs JE, Paddock CD, 2003. The ascendancy of Amblyomma americanum as a vector of pathogens affecting humans in the United States. Annu Rev Entomol 48: 307-337.

22. Hamilton KS, Standaert SM, Kinney MC, 2004. Characteristic peripheral blood findings in human ehrlichiosis. Mod Pathol 17: 512-517.

23. Lockhart JM, Davidson WR, Stallknecht DE, Dawson JE, Howerth E, 1997. Isolation of Ehrlichia chaffeensis from wild white-tailed deer (Odocoileus virginianus) confirms their role as natural reservoir hosts. J Clin Microbiol 35: 1681-1686.
24. Stafford KC, 3rd, Molaei G, Little EAH, Paddock CD, Karpathy SE, Labonte AM, 2018. Distribution and establishment of the lone star tick in Connecticut and implications for range expansion and public health. J Med Entomol 55: 1561-1568.

25. Dumler JS, Chen SM, Asanovich K, Trigiani E, Popov VL, Walker $\mathrm{DH}, 1995$. Isolation and characterization of a new strain of Ehrlichia chaffeensis from a patient with nearly fatal monocytic ehrlichiosis. J Clin Microbiol 33: 1704-1711.

26. Paddock C, Sumner J, Shore G, Bartley D, Elie R, McQuade J, Martin C, Goldsmith C, Childs J, 1997. Isolation and characterization of Ehrlichia chaffeensis strains from patients with fatal ehrlichiosis. J Clin Microbiol 35: 2496-2502.

27. Standaert SM, Yu T, Scott MA, Childs JE, Paddock CD, Nicholson WL, Singleton JA, Jr., Blaser MJ, 2000. Primary isolation of Echrlichia chaffeensis from patients with febrile illnesses: clinical and molecular characteristics. J Infect Dis 181: 1082-1088.

28. Cheng C, Ganta RR, 2008. Laboratory maintenance of Ehrlichia chaffeensis and Ehrlichia canis and recovery of organisms for molecular biology and proteomics studies. Curr Protoc Microbiol Chapter 3: Unit 3A 1.

29. Kumar S, Stecher G, Tamura K, 2016. MEGA7: molecular evolutionary genetics analysis version 7.0 for bigger datasets. $\mathrm{Mol}$ Biol Evol 33: 1870-1874.

30. Cheng C, Paddock CD, Reddy Ganta R, 2003. Molecular heterogeneity of Ehrlichia chaffeensis isolates determined by sequence analysis of the 28-kilodalton outer membrane protein genes and other regions of the genome. Infect Immun 71: 187-195.

31. Paddock CD et al., 2001. Infections with Ehrlichia chaffeensis and Ehrlichia ewingii in persons coinfected with human immunodeficiency virus. Clin Infect Dis 33: 1586-1594.

32. Nelder MP, Russell CB, Clow KM, Johnson S, Weese JS, Cronin K, Ralevski F, Jardine CM, Patel SN, 2019. Occurrence and distribution of Ambylomma americanum as determined by passive surveillance in Ontario, Canada (1999-2016). Ticks Tick-Borne Dis 10: 146-155.

33. Williams-Newkirk AJ, Burroughs M, Changayil SS, Dasch GA, 2015. The mitochondrial genome of the lone star tick (Amblyomma americanum). Ticks Tick-Borne Dis 6: 793-801.

34. Webb SL, Hewitt DG, Hellickson MW, 2007. Scale of management for mature male white-tailed deer as influenced by home range and movements. J Wildl Manag 71: 1507-1512.

35. Wigley TB, Sweeney JM, Garner ME, Melchiors MA, 1986. Wild turkey home ranges in the Ouachita mountains. J Wildl Manag 50: 540-544.

36. Litvaitis JA, Shaw JH, 1980. Coyote movements, habitat use, and food habits in southwestern Oklahoma. J Wildl Manag 44: 62-68.

37. Machado RZ, Andre MR, Werther K, de Sousa E, Gavioli FA, Alves Junior JR, 2012. Migratory and carnivorous birds in Brazil: reservoirs for Anaplasma and Ehrlichia species? Vector Borne Zoonotic Dis 12: 705-708.

38. Erwin JA, Fitak RR, Dwyer JF, Morrison JL, Culver M, 2016. Molecular detection of bacteria in the families Rickettsiaceae and Anaplasmataceae in northern crested caracaras (Caracara cheriway). Ticks Tick Borne Dis 7: 470-474.

39. Hamer SA, Goldberg TL, Kitron UD, Brawn JD, Anderson TK, Loss SR, Walker ED, Hamer GL, 2012. Wild birds and urban ecology of ticks and tick-borne pathogens, Chicago, Illinois, USA, 2005-2010. Emerg Infect Dis 18: 1589-1595.

40. Alekseev AN, Dubinina HV, Van De Pol I, Schouls LM, 2001. Identification of Ehrlichia spp. and Borrelia burgdorferi in ixodes ticks in the baltic regions of Russia. J Clin Microbiol 39: 2237-2242.

41. Durden LA, Oliver J, James H, Kinsey AA, 2001. Ticks (Acari: Ixodidae) and spirochetes (Spirochaetaceae: Spirochaetales) recovered from birds on a Georgia barrier island. JMed Entomol 38: 231-236.

42. Kuo CC, Lin YF, Yao CT, Shih HC, Chung LH, Liao HC, Hsu YC, Wang HC, 2017. Tick-borne pathogens in ticks collected from birds in Taiwan. Parasit Vectors 10: 587.

43. Alekseev AN, Dubinina HV, Semenov AV, Bolshakov CV, 2001. Evidence of ehrlichiosis agents found in ticks (Acari: Ixodidae) collected from migratory birds. J Med Entomol 38: 471-474. 